Note

\title{
Prediction of Salmonella Enteritidis Growth in Pasteurized and Unpasteurized Liquid Egg Products with a Growth Model
}

\author{
MOHAMMAD ZAHER SAKHA ${ }^{1}$, AND HIROSHI FUJIKAWA ${ }^{1,2 *}$ \\ 'Department of Applied Veterinary Science, The Graduated School of \\ Veterinary Sciences, Gifu University \\ 2 Laboratory of Veterinary Public Health, Faculty of Agriculture, Tokyo University of \\ Agriculture and Technology, 3-5-8 Saiwai-cho, Fuchu, Tokyo 183-8509, Japan
}

Received 2 July, 2012/Accepted 27 September, 2012

\begin{abstract}
Growth prediction of a four-strain cocktail of Salmonella Enteritidis in commercial products of pasteurized and unpasteurized liquid whole egg was studied with the new logistic model that we developed. The growth data of the pathogen in the liquid egg products at constant temperatures in our recent study (Sakha and Fujikawa, Biocont. Sci., 2012) were used for prediction. With estimated values of the parameters in the model, it successfully predicted the Salmonella growth in the liquid egg products at dynamic temperature conditions in the high and low ranges. The Baranyi model, which is well known worldwide, could predict Salmonella growth in the pasteurized product at the dynamic temperature conditions in the high range only. This study would be, in our knowledge, the first report on the prediction of Salmonella growth in both pasteurized and unpasteurized liquid egg products at dynamic temperature conditions with a mathematical model.
\end{abstract}

Key words : Salmonella Enteritidis / Dynamic temperature / Liquid egg/Growth prediction / Logistic model.

People are consuming an ever increasing amount of commercial liquid egg products worldwide (FAO, 2010). Liquid egg products are divided into three types according to the contents, including whole egg, egg yolk, and albumen. Also, they consist of pasteurized and unpasteurized products. Pasteurized liquid egg products are made by heating raw liquid eggs at a given temperature to kill Salmonella, which may contaminate shell eggs. Especially, the Salmonella serotype Enteritidis, which sometimes contaminates shell eggs, has caused many food poisoning outbreaks worldwide and is one of the most frequently implicated Salmonella serotypes in food poisoning cases (Patrick et al., 2004). Hara-Kudo and Takatori (2009) reported that $73.5 \%$ of unpasteurized liquid egg products and as high as $1.7 \%$ of pasteurized

*Corresponding author. Tel/Fax: +81-42-367-5598

E-mail: fujik (a)cc.tuat.ac.jp products in Japan were contaminated with Salmonella. McQuestin et al. (2010) described that a greater biosecurity risk is associated with coldstored, liquid egg products that are consumed uncooked or "lightly cooked", typically as ingredients in meringue, homemade ice cream, and so on. Therefore, information on the growth kinetics of Salmonella in both pasteurized and unpasteurized liquid egg products is very important to prevent food poisoning outbreaks originating from liquid egg products contaminated with Salmonella.

There are several models for microbial growth such as the Baranyi model, the modified Gompertz model, and the three-phase linear model that are known worldwide (Baranyi and Roberts, 1994; Buchanan et al., 1997; Gibson et al., 1987). Among them the Baranyi model has been the most well-known and widely used worldwide.

We also developed a growth model called the new 
logistic (NL) model from the original logistic model (Fujikawa et al., 2003). It has been shown to successfully describe and predict growth curves of Escherichia coli, Salmonella, and Staphylococcus aureus in sterile foods or media (Fujikawa et al., 2003, 2004; Fujikawa and Morozumi, 2005, 2006). The differential form of the model is described in Eq. 1 (Fujikawa and Morozumi, 2005).

$$
\frac{d N}{d t}=r N\left\{1-\left(\frac{N}{N_{\max }}\right)^{m}\right\}\left\{1-\left(\frac{N_{\min }}{N}\right)^{n}\right\}
$$

Here $N$ is the population of a microorganism (CFU/g) at time $t(h) . r$ is the rate constant of growth, or the maximum specific growth rate $(1 / \mathrm{h}) . N_{\max }$ and $N_{\min }$ correspond to the maximum and the initial populations (CFU/g), respectively. Parameters $m$ and $n$ $(>0)$ are related to the curvature of the deceleration phase and the period of the lag phase, respectively. The NL model is solved numerically with the 4th-order Runge-Kutta method (Fujikawa et al., 2003, 2004; Fujikawa and Morozumi, 2005, 2006).

On the other hand, food materials and commercially available unsterilized food products are contaminated with natural microflora (NM). Competition between a pathogen and NM in a food would influence the growth of the pathogen in foods. Recently the kinetics of microbial competition in food has attracted the interest of many researchers. We also succeeded in describing and predicting the growth patterns of a Salmonella Enteritidis strain and NM in raw ground chicken with our growth model (Zaher and Fujikawa, 2011).

Recently we studied the kinetics of Salmonella Enteritidis growth in pasteurized and unpasteurized liquid whole egg products with the NL model (Sakha and Fujikawa, 2012). The model precisely described the Salmonella growth in the products at various initial concentrations and at constant temperatures and also characterized the growth kinetics of the pathogen in the products with the values for the parameters in the model.

McQuestin et al. (2010) reported on the kinetics of growth of the Salmonella Typhimurium DT104 in pasteurized liquid egg products. However, there seem to be, to our knowledge, no systematic studies on the prediction of Salmonella growth in pasteurized and unpasteurized liquid egg products. Therefore, in the present study we examined if the NL model could predict the Salmonella Enteritidis growth in the above products at dynamic temperatures, because the temperature of a liquid egg product which might be contaminated with Salmonella would change through the periods of transportation and storage. We also examined the prediction by using the Baranyi model for comparison in the present study, as we analyzed the Salmonella growth at the constant temperatures with the model (Sakha and Fujikawa, 2012).

The materials and methods used in the present study were the same as those in our recent study (Sakha and Fujikawa, 2012). Briefly, four Salmonella Enteritidis strains SE2, SE3, SE5, and 04-137, which were kindly provided by the Tokyo Metropolitan Research Institute of Public Health, Japan, were activated on XLD agar (Oxoid, Basingstoke, UK). Cells of well-grown colonies on the plates were incubated in Trypticase soy broth (Oxoid) with shaking. Cultured cells ( $1 \mathrm{ml}$ each) of the strains were washed with saline and then mixed together. The mixture was diluted with saline to a cell suspension of $10^{5}$ $\mathrm{CFU} / \mathrm{ml}$.

The pasteurized $\left(60{ }^{\circ} \mathrm{C}\right.$ for $\left.3.5 \mathrm{~min}\right)$ and unpasteurized whole liquid egg products that were used in our recent study (Sakha and Fujikawa, 2012) were also used for the experiments described herein. The liquid egg products were stored in sterile plastic cups at $-30^{\circ} \mathrm{C}$ until use and then thawed at $<10^{\circ} \mathrm{C}$ overnight for use.

Liquid egg samples were spiked with the Salmonella cell suspension prepared above (2 $\mathrm{ml} / 100 \mathrm{~g}$ ) and thoroughly mixed. Portions (10 g) were then placed in sterile glass bottles $(110 \mathrm{ml})$ with caps. The glass bottles were stored in a programmable incubator (SU-221; Espec Co., Osaka, Japan) at dynamic temperatures. Immediately after incubation, each sample (three bottles per data point) was taken from the incubator and cooled. The sample temperature was measured in duplicate every $30 \mathrm{~s}$ throughout the experiment with a digital thermometer (AM-7002; Anritsu Meter Co., Tokyo).

After $10 \%$ food homogenates were prepared for the samples with a buffered sodium chloride peptone solution, 10 -fold dilutions $(0.1 \mathrm{ml}$ each) of the homogenates were placed on standard method agar plates (Nissui Pharmaceuticals, Tokyo, Japan) and XLD agar plates (Oxoid) in duplicate for the counting of viable bacteria and Salmonella, respectively. After incubation, colonies suspected to be Salmonella were examined for identification with a serological test using the antiserum to Salmonella $O$ antigens (Denka-Seiken, Tokyo). The average bacterial number on two plates at a certain dilution for each sample was calculated. Average bacterial counts for each data point in triplicate were then calculated.

Performance of the model for each temperature pattern was evaluated with ( $i$ ) the mean of the square error, MSE, between log-transformed cell concentrations estimated with the model $\left(\log N_{\text {est }}\right)$ and those 
observed $\left(\log N_{\text {obs }}\right)$ at the observation points (Fujikawa et al., 2004; Fujikawa and Morozumi, 2005, 2006; Juneja et al., 2007; Zaher and Fujikawa, 2011) and (ii) the residual, which is the value of $\log N_{\text {obs }}$ minus $\log N_{\text {est }}$, for each observation point during the growth (Oscar, 2009).

Growth prediction of Salmonella Enteritidis in the liquid egg products at a dynamic temperature with the $\mathrm{NL}$ model was performed by introducing the values for $r$ and $N_{\max }$ and parameters $m$ and $n$, which were estimated at constant temperatures, into the model (Eq. 1) (Fujikawa et al., 2003, 2004; Fujikawa and Morozumi, 2005, 2006). Here $T\left({ }^{\circ} \mathrm{C}\right)$ was the temperature at time $t$. The value for $r$ at $T$ was estimated with Eqs. 2 and 3 in the pasteurized and unpasteurized products, respectively (Sakha and Fujikawa, 2012).

$$
\begin{aligned}
& \sqrt{r}=0.0473(T-4.02) \\
& \sqrt{r}=0.0313(T-4.48)
\end{aligned}
$$

The value for $N_{\max }$ in the pasteurized product at $T$ was set to be $10^{9.7} \mathrm{CFU} / \mathrm{g}$, the average at the constant temperature from 8 to $36{ }^{\circ} \mathrm{C}$ (Sakha and Fujikawa, 2012). The $N_{\max }$ value at $T$ in the unpasteurized product was expressed with Eq. 4 (Sakha and Fujikawa, 2012).

$$
N_{\text {max }}=10^{0.906 T+2.92}
$$

For $m$ and $n$ in the NL model, the averages for the parameters obtained at the constant temperatures were applied for prediction (0.42 and 4.9 in the pasteurized product and 1.5 and 11.5 in the unpasteurized, respectively) (Fujikawa et al., 2004; Fujikawa and Morozumi, 2005, 2006; Fujikawa and Kano, 2009).

With the values for $r$ and $N_{\max }$ estimated with Eqs. 24 and the above parameter values, the NL model succeeded in predicting the growth of Salmonella in both liquid egg products stored at various dynamically changing temperatures (Figs. $1 \mathrm{~A}, \mathrm{~B}$ and $2 \mathrm{~A}, \mathrm{~B}$ ). Here, the dynamic temperature patterns included (A) high and (B) low temperature ranges. Values of MSE in log units for those predictions in the figures were very small, being $0.052(1 \mathrm{~A}), 0.13(1 \mathrm{~B}), 0.041(2 \mathrm{~A})$, and $0.014(2 \mathrm{~B})$. The residuals between the observed and predicted populations (in log) for the points in the growth curves in Figs. 1 and 2 were plotted along the time (Fig. 3). The acceptable rates in the range of +0.5 and -1.0 log for all data points were $100 \%$
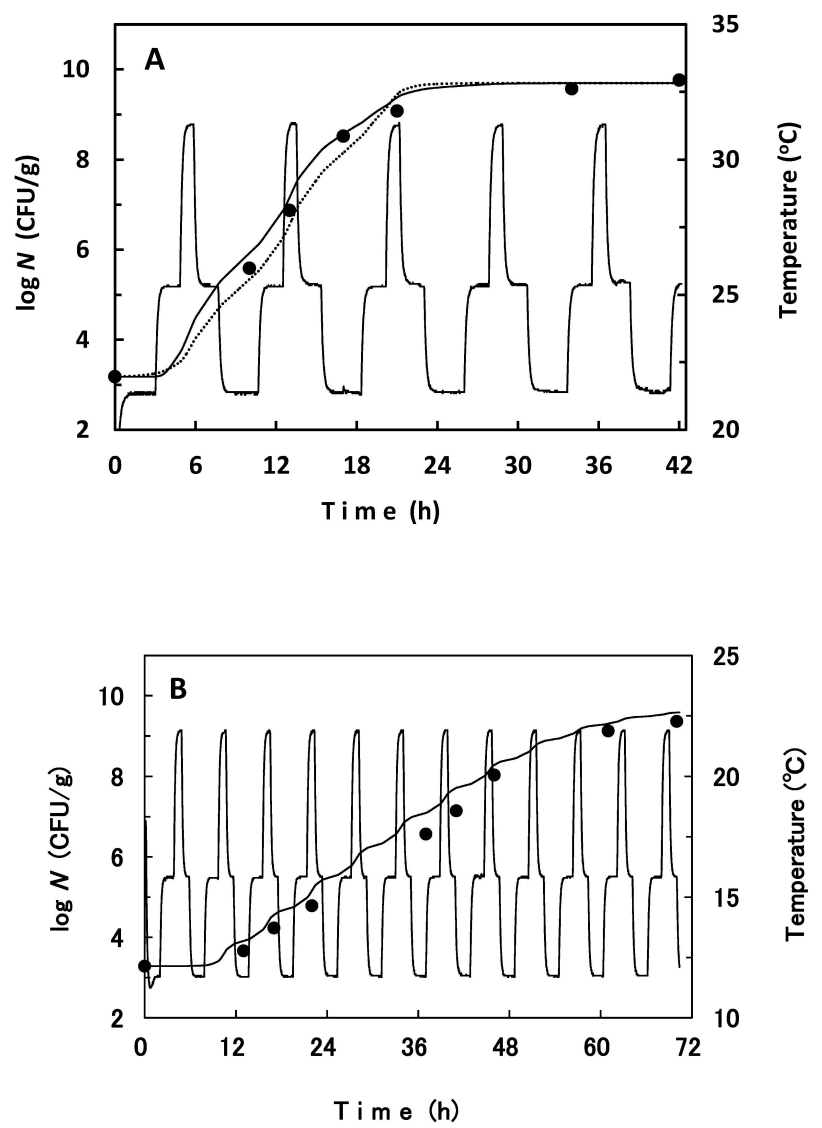

FIG. 1. Prediction of Salmonella Enteritidis growth in pasteurized liquid egg at dynamic temperature patterns in the high range $(A)$ and the low range $(B)$. Closed circles are the measured Salmonella population. Bars indicate SDs at data points, but they are too short to show in the figures. Growth curves are predicted from the measured temperatures (regularly changing curves) during the storage period with the NL model (solid lines) and the Baranyi model (a dotted line). Regularly changing curves during the storage period are the measured temperature of the product.

(27/27), which were considerably over the level of acceptance of $70 \%$ (Oscar, 2009). When a narrower acceptable range was set between +0.5 and -0.5 log, a proposition as high as $92.6 \%(25 / 27)$ was still within the acceptable range. The average of the residuals for all points was $-0.138 \mathrm{log}$, meaning that the observed populations were slightly smaller than the predicted ones. These results showed that the NL model successfully predicts the Salmonella growth in the liquid egg products at the changing temperatures. This prediction for Salmonella growth in unpasteurized products at dynamic temperatures would be the first of its type to our knowledge.

We could not predict the growth of NM in the unpasteurized liquid egg products with the NL model in this study, while we could do so for the NM in the raw ground chicken (Zaher and Fujikawa, 2011). 

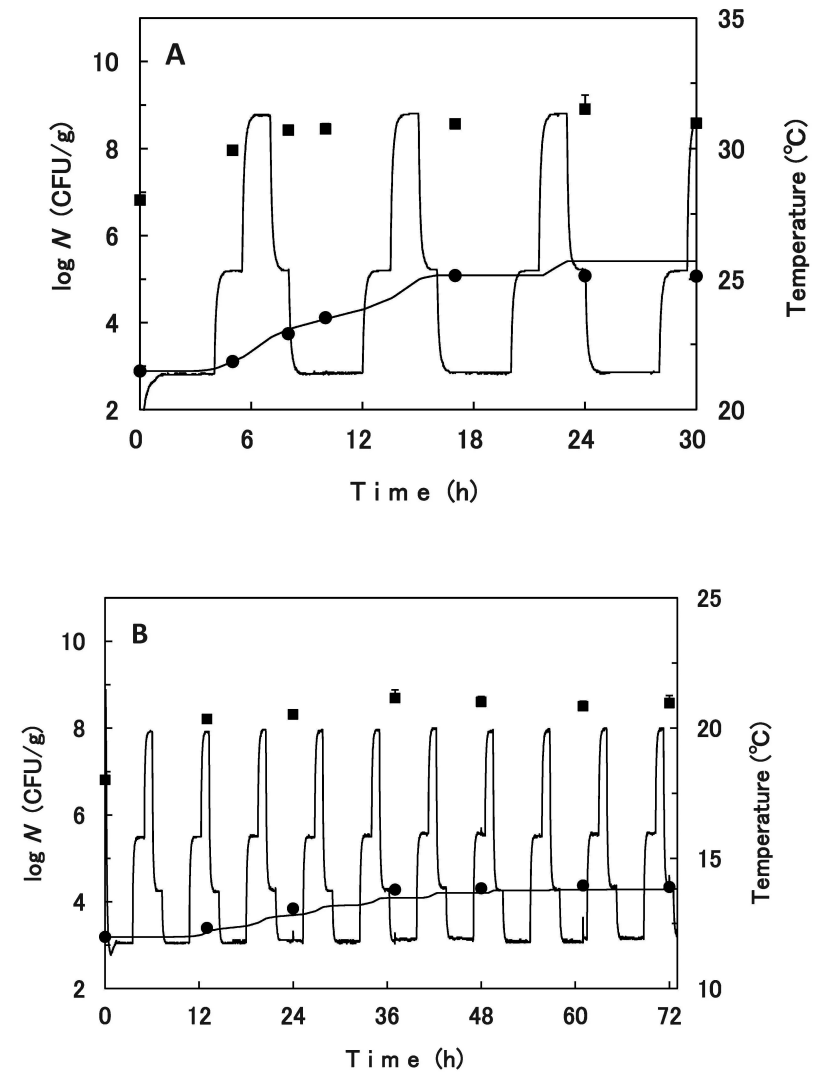

FIG. 2. Prediction of Salmonella Enteritidis growth in unpasteurized liquid egg at dynamic temperature patterns in the high range $(A)$ and the low range (B). Closed circles and squares are the measured populations of Salmonella and NM, respectively. Bars indicate SDs at data points. Growth curves are predicted with the NL model. Regularly changing curves during the storage period are the measured temperatures of the product.

This came from the fact that the growth data of NM at the constant temperatures in the liquid egg could not be analyzed with the NL model, because the initial level of NM in the product was too high to make a sigmoidal growth curve during the incubation (Sakha and Fujikawa, 2012). If the initial level of NM of the liquid egg had been low enough to show a sigmoidal curve, the model could have predicted the NM growth.

For comparison, the Baranyi model was also examined for its capacity to predict Salmonella growth at the dynamic temperatures in the two types of liquid egg products. The model is built under the prerequisite condition that the (numerical) products of the maximum specific growth rate $(1 / h)$ and the lag period (h) in the growth curves of a given microorganism under various conditions should be constant (Baranyi and Roberts, 1994). With these values, one can predict microbial growth under a new condition

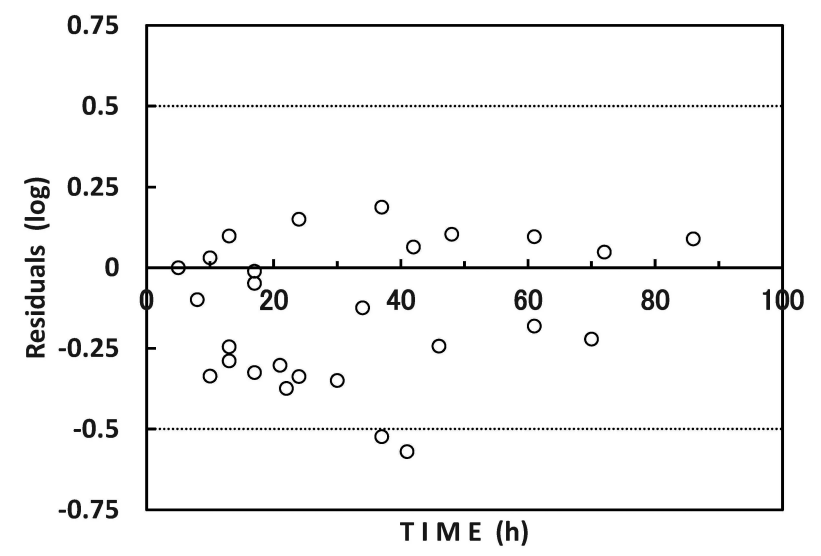

FIG. 3. Residual plots for the populations by the NL model along through the storage period. All residuals between observed and estimated values shown in Figs. 1 and 2 are plotted. Dotted lines show the boundaries of acceptance.

with this model (Baranyi and Roberts, 1994).

Thus we first estimated the numerical products for the Salmonella growth curves in the liquid egg samples at the constant temperatures ranging from 8 to $36{ }^{\circ} \mathrm{C}$ examined in our recent study (Sakha and Fujikawa, 2012). The values for the maximum specific growth rate and the lag period for the growth curves were estimated with a computer program, DMFit (www.ifr.ac.uk/safety/DMFit), which analyzes microbial growth data with the Baranyi model. Consequently, the products at the constant temperatures were constant in neither liquid egg product; the values were lower at the lower temperatures (data not shown). The reasons for this tendency were not understood. However, the values in the temperature range between 20 and $36{ }^{\circ} \mathrm{C}$ in the pasteurized liquid egg were relatively constant; the average for the value in this range was estimated to be $3.34 \pm 0.516$. Thus, with this average, we predicted the Salmonella growth at the dynamic temperature pattern of the higher range in the present study with the Baranyi model. As a result, the model could well predict the bacterial growth at a changing temperature (Fig. 1A). The MSE value $(0.075 \mathrm{log})$ was small, but a little higher than that by the NL model $(0.052 \mathrm{log})$. These results suggested that the Baranyi model could be applied for the growth prediction in a limited temperature range where the numeral product is relatively constant.

This study showed that the NL model could well predict Salmonella growth in the pasteurized and unpasteurized liquid egg products under dynamic temperature conditions, similar to the Salmonella growth in raw ground chicken with NM (Zaher and Fujikawa, 2011). These prediction results should be applied to food safety measures to prevent the 
occurrence of food poisoning outbreaks by the pathogen. We have developed programs to predict the growth of microbes including Escherichia coli, Staphylococcus aureus, Vibrio parahaemolyticus at dynamic temperatures using Microsoft Excel (Fujikawa et al., 2006, 2009). Therefore, we would like to develop a new prediction program for the Salmonella growth in those food materials using Microsoft Excel in the near future. The program would be a useful tool for those concerned to estimate the bacterial growth in such food materials at the temperature history they measure.

\section{ACKNOWLEDGMENTS}

This study was supported by Kieikai Research Foundation. The authors thank Dr. N. Sashihara for providing us with the liquid egg products and useful suggestions.

\section{REFERENCES}

Baranyi, J., and Roberts, T.A. (1994) A dynamic approach to predicting bacterial growth in food. Int. J. Food Microbiol., 23, 277-294.

Buchanan, R.L., Whiting, R.C., and Damert, W.C. (1997) When is simple good enough: a comparison of the Gompertz, Baranyi, and three-phase linear models for fitting bacterial growth curves. Food Microbiol., 14, 313326.

FAO (Food and Agriculture Organization) (2010) Poultry meat and eggs. http://www.fao.org/docrep/012/ al175e/al175e.pdf " > http://www.fao.org/docrep/012/ al175e/al175e.pdf. Accessed 25 June 2012.

Fujikawa, H., Kai, A., and Morozumi, S. (2003) A new logistic model for bacterial growth. J. Food Hyg. Soc. Japan, 44, 155-160.

Fujikawa, H., Kai, A., and Morozumi, S. (2004) A new logistic model for Escherichia coli at constant and dynamic temperatures. Food Microbiol., 21, 501-509.

Fujikawa, H., and Morozumi, S. (2005) Modeling surface growth of Escherichia coli on agar plates. Appl. Environ.
Microbiol., 71, 7920-7926.

Fujikawa, H., and Morozumi, S. (2006) Modeling Staphylococcus aureus growth and enterotoxin production in milk. Food Microbiol., 23, 260-267.

Fujikawa, H., Yano, K., Morozumi, S., Kimura, B., and Fujii, T. (2006) Development of a microbial growth prediction program at various temperature patterns. (in Japanese) J. Food Hyg. Soc. Japan. 47, 288-292.

Fujikawa, H., and Kano, Y. (2009) Development of a program to fit data to a NL NL model for microbial growth. Biocont. Sci., 14, 83-86.

Fujikawa, H., Kimura, B., and Fujii, T. (2009) Development of a predictive program for Vibrio parahaemoliticus growth under various environmental conditions. Biocont. Sci. 14, 127-131.

Gibson, A.M., Bratchell, N., and Roberts, T.A. (1987) The effect of sodium chloride and temperature on the rate and extent of growth of Clostridium botulinum type $A$ in pasteurized pork slurry. J. Appl. Bacteriol., 62, 479-490.

Hara-Kudo, Y., and Takatori, K. (2009) Microbial quality of liquid egg and Salmonella infection status in Japan. J. Food Hyg. Soc. Japan, 60, 34-40.

Juneja. V.K., Melendres, M.V., Huang, L., Gumudavelli, V., Subbiah, J., and Thippareddi, H. (2007) Modeling the effect of temperature on growth of Salmonella in chicken. Food Microbiol., 24, 328-335.

McQuestin, O.J., Musgrove, M.T., and Tamplin, M.L. (2010) Kinetics of growth and inactivation of Salmonella enterica serotype Typhimurium DT104 in pasteurized liquid egg products. Food Microbiol., 27, 396-402.

Oscar, T.P. (2009) General regression neural network and Monte Carlo simulation model for survival and growth of Salmonella on raw chicken skin as a function of serotype, temperature, and time for use in risk assessment. J. Food Prot., 72, 2078-2087.

Patrick, M.E., Adcock, P.M., Gomez, T.M., Altekruse, S.F., Holland, B.H., Tauxe, R.V., and Swerdlow, D.L. (2004) Salmonella Enteritidis infections, United States, 19851999. Emerg. Infect. Dis., 10, 1-7.

Zaher, S.M., and Fujikawa, H. (2011) Effect of NM on the growth kinetics of Salmonella Enteritidis strain 04-137 in raw ground chicken., J. Food Prot., 74, 735-742.

Sakha, M. Z., and Fujikawa, H. (2012) Growth characteristics of Salmonella Enteritidis in pasteurized and unpasteurized liquid egg products Biocont. Sci., 17, 183190. 